\title{
NUEVO GÉNERO Y NUEVA ESPECIE DE PARAJAPYGIDAE (HEXAPODA: DIPLURA) DE LA SELVA LACANDONA, MÉXICO
}

\begin{abstract}
Arturo GarCíA-Gómez
Ecología y Sistemática de Microartrópodos, Depto. de Ecología y Recursos Naturales, Facultad de Ciencias, Universidad Nacional Autónoma de México. 04510 D.F. MEXICO. gab12y@yahoo.com.mx

García-Gómez, A. 2009. Nuevo género y nueva especie de Parajapygidae (Hexapoda: Diplura) de la Selva Lacandona, México. Acta Zoológica Mexicana (n. s.), 25(3): 527-535.

RESUMEN. Se describe un nuevo género y una nueva especie de la familia Parajapygidae de la selva Lacandona, Chiapas, tomando en cuenta el cerco terminal de tipo asimétrico con cuatro dientes internos como carácter diagnóstico del nuevo género.
\end{abstract}

Palabras clave: Parajapygidae, Lacandonajapyx, México, Chiapas, Selva Lacandona.

García-Gómez, A. 2009. New genus and new species of Parajapygidae (Hexapoda: Diplura) from Lacandon jungle, México. Acta Zoológica Mexicana (n. s.), 25(3): 527-535.

ABSTRACT. The description of a new genus and new species of the family Parajapygidae from Lacandon jungle of Chiapas is given. The asymmetric terminal forceps with four internal teeth as the diagnostic characters of new genus.

Key words: Parajapygidae, Lacandonajapyx, México, Chiapas, Lacandon jungle.

\section{INTRODUCCIÓN}

La familia Parajapygidae (Hexapoda: Diplura) fue establecida en 1959 por Pagés con la especie tipo Parajapyx isabellae (Paclt 1957), de acuerdo con la ausencia de palpos labiales y la presencia de dos estigmas toráxicos (Pagés 1989). Posteriormente Plactl (1957) especificó más características, como los apéndices laterales subcoxales en ambos sexos, empodio formando una uña media (unguiculus), y los cercos simétricos. Por último Ferguson (1990) consideró también la presencia de un orificio glandular cerca de la superficie de los cercos.

La familia se divide en tres géneros: Miojapyx, Ectasjapyx y Parajapyx. El género Miojapyx está distribuido al sur de México y tiene como carácter principal, vesículas coxales en los esternitos abdominales. El género Ectasjapyx es endémico de Angola y presenta una mandíbula con 4 dientes y 3 dentículos entre ellos. El género Parajapyx es cosmopolita y tiene una mandíbula de 5 dientes y 4 dentículos (Silvestri 1929 y Paclt 1957).

Recibido: 16/01/2009; aceptado: 11/06/2009. 


\section{MATERIAL Y MÉTODOS}

Se colectaron cinco ejemplares, todos ellos hembras, en la reserva comunal de la Sierra de Corolita en la Selva Lacandona, Chiapas, México. Las cuales se montaron en preparaciones semipermanentes con líquido de Hoyer. Las mediciones en milímetros se tomaron con una reglilla ocular micrométrica en un microscopio de contraste de fases con cámara clara Carl Zeiss ${ }^{\circledR}, 4746$ 20-9900, y objetivos 40/0.65 y $100 / 0.65$.

Para la descripción se utilizaron las siguientes abreviaturas: $\mathrm{n}=$ normales, $\mathrm{m}=$ microseda, $\mathrm{M}=$ macroseda, cuya posición en los distintos tergos se indica como: $\mathrm{ma}=$ media anterior, $\mathrm{la}=$ latero anterior, $\mathrm{mp}=$ media posterior $\mathrm{y} \mathrm{lp}=$ lateral posterior; $\mathrm{SB}=$ sensila baciliforme, $\mathrm{SP}=$ sensila placoide, $\mathrm{LC}=$ lacinia. Para los nombres de las placas dorsales se utilizó la nomenclatura propuesta por Pagés (1996).

\section{Lacandonajapyx gen. nov.}

Diagnosis. Familia Parajapygidae, similar al género Parajapyx; 18 segmentos antenales, sensilas ausentes en los segmentos I-IV, mandíbula con cinco dientes, el segundo y cuarto de menor tamaño, este último bífido. Entre los dientes se presentan tres dentículos; maxila con cinco lamelas, la primera lisa, las restantes pectinadas, subcoxa con dos líneas de sedas simples de igual tamaño, las anteriores en número que las posteriores, estilos típicos de Parajapyx, segmento VIII diferente en forma y tamaño del IX, cercos unisegmentados con forma de pinzas, sin poro glandular. Propuesta del género, número de dentículos y lamelas, al ser uno menos y tres más, respectivamente, así como la ausencia de poro glandular cerca de los cercos, en relación al género cercano.

Especie tipo: Lacandonajapyx cristinae sp. nov.

Etimología. El nombre corresponde a la localidad tipo: Lacandona, Selva Lacandona y japyx del nombre genérico. Género es femenino.

\section{Lacanjapyx cristinae sp.nov.}

(Fig. 1-15)

Descripción. Holotipo (우 ): longitud del cuerpo $2.71 \mathrm{~mm}$, sin antenas ni cercos. Paratipos (4우우 adultas) longitud $(\mathrm{n}=5) 2.71 \mathrm{~mm}$, rango 2.70- $2.72 \mathrm{~mm}$, tegumento liso, sin ornamentaciones.

Cabeza. Vertex: con $18+19$ sedas (Fig. 1), frente sin sedas, clípeo con 1+1 seda simple, el labro presenta dos sedas medias, $6+6 \mathrm{~m}$ y $1+1 \mathrm{SB}$ en la parte más externa (Fig. 2). Centralmente: Lóbulo interno, $1+1 \mathrm{~m}$; lóbulo externo, 9+9 sedas; coxa, 2+2 sedas y $2+2 \mathrm{~m}$; sin palpo labial, en su lugar $1 \mathrm{M}$ acompañada de dos sedas; admento, una $\mathrm{M}$ además de sedas normales; pli oral, 6-8+5-7 sedas, submento, $2+2$ sedas. 
Estructuras bucales. Lámina distal de la lacinia falciforme, posteriormente presenta cinco laminas, la primera (distal) lisa y restantes pectinadas; galea simple no esclerosada; mandíbula con cinco dientes y tres dentículos entre ellos; palpo maxilar con 9 sedas además de $1 \mathrm{~m}$ (Fig. 3).

Antenas. Con 18 artejos; artejo I, seis microsedas; I-IV, sin tricobotrias; V, con dos SB (en la figura 4, sólo se muestra 1); VI-VII, tres; IX-XVI, cuatro; XVII, seis; XVIII, ocho y cuatro sensilas placoides (en la figura 5 sólo se aprecian 4 y 2, respectivamente).

Tórax (Fig. 6). La distribución de las sedas del tórax se muestra en el Cuadro 1.

Cuadro 1. Distribución de las sedas torácicas de L. cristinae nov. sp.

\begin{tabular}{|c|c|c|c|c|c|c|}
\hline & \multirow[b]{2}{*}{$\mathbf{m}$} & \multicolumn{4}{|c|}{$\mathbf{M}$} & \multirow[b]{2}{*}{$\mathbf{n}$} \\
\hline & & ma & $\mathbf{m p}$ & la & lp & \\
\hline Pronoto & $4+4$ & & $1+1$ & $1+1$ & & $5+5$ \\
\hline Prescuto & $6+6$ & & & & & $1+1$ \\
\hline Mesoescuto & & & $1+1$ & $2+2$ & $1+1$ & $7+7$ \\
\hline Prescuto & $7+7$ & & & & & $2+2$ \\
\hline Metaescuto & $1+1$ & & $1+1$ & $2+2$ & $1+1$ & $7+7$ \\
\hline
\end{tabular}

Pata III (Fig. 7). Longitud $0.2 \mathrm{~mm}$, con sedas lisas y sin M; Coxa, $2 \mathrm{~m}$ y $3 \mathrm{n}$; trocánter, $1 \mathrm{~m}$ ventral, $3 \mathrm{n}$ dorsales; fémur $10 \mathrm{n}$; tibia $7 \mathrm{n}$; el trocánter $9 \mathrm{n}$; uñas simétricas, con unguiculo y sin seda pretarsal (Fig. 8).

Abdomen. La distribución de las sedas dorsales se muestra en el Cuadro 2.

Cuadro 2. Distribución de las sedas abdominales de L. cristinae nov. sp.

\begin{tabular}{|c|c|c|c|c|c|c|c|}
\hline & & \multirow[b]{2}{*}{$\mathbf{m}$} & \multicolumn{4}{|c|}{$\mathbf{M}$} & \multirow[b]{2}{*}{$\mathbf{n}$} \\
\hline & & & ma & $\mathbf{m p}$ & la & lp & \\
\hline I & Prescuto & $3+3$ & & & & & $2+2$ \\
\hline I & Escuto & $3+3$ & $1+1$ & $1+1$ & $1+1$ & $1+1$ & $5+5$ \\
\hline II-VII & Prescuto & $4+4$ & & & & & $1+1+1$ \\
\hline II-VII & Escuto & $4+4$ & $1+1$ & $1+1$ & $3+3$ & $1+1$ & $6+6$ \\
\hline VIII & & $7+7$ & $1+1$ & $1+1$ & $2+2$ & $2+2$ & $5+5$ \\
\hline IX & & $5+5$ & $1+1$ & & $1+1$ & & $2+2$ \\
\hline$X$ & & & $1+1+1$ & $1+1$ & $1+1$ & $1+1$ & $2+2$ \\
\hline
\end{tabular}



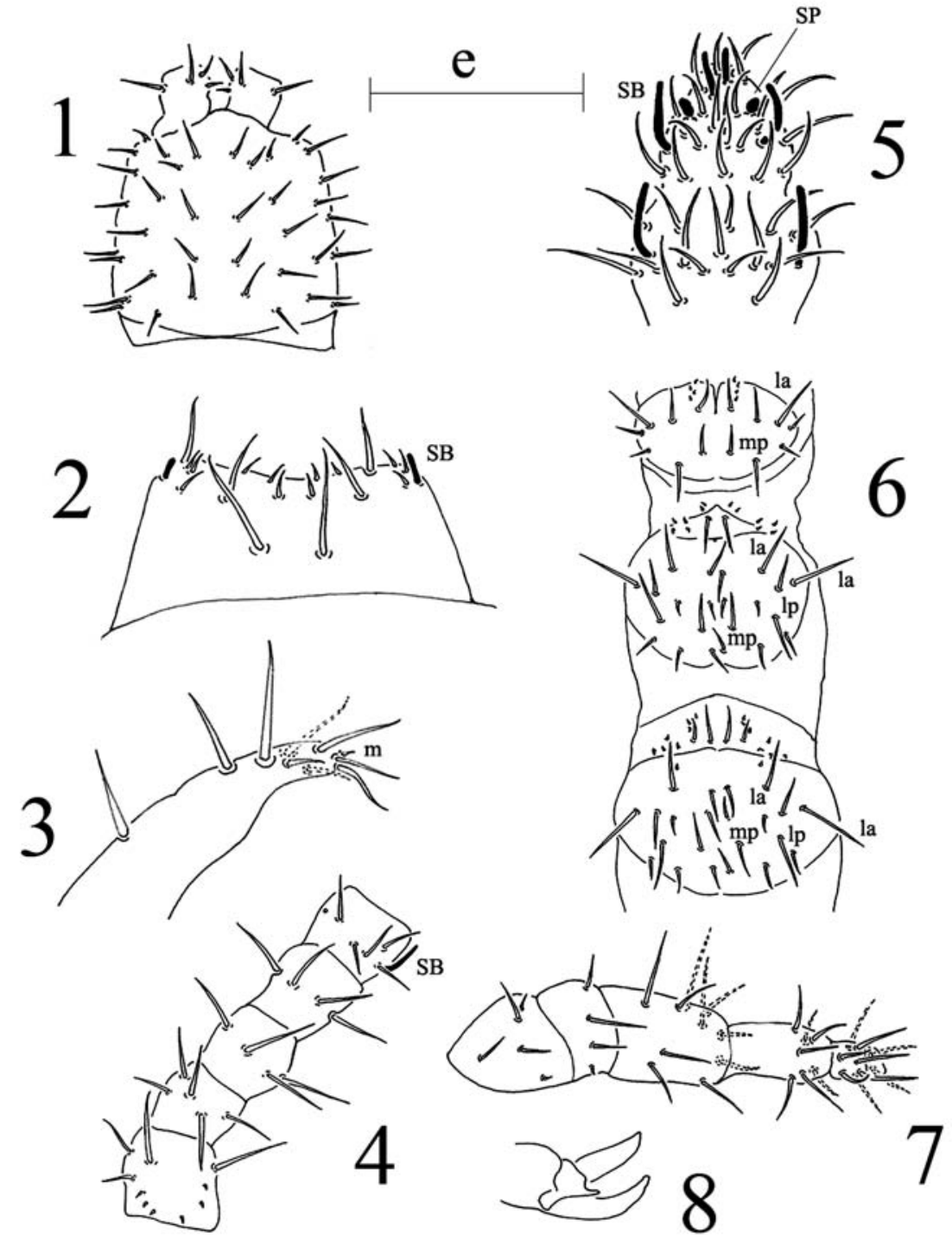

Figuras 1-8. Lacandonajapyx cristinae nov. sp. 1, Cabeza; 2, labro; 3, Palpo labial; 4, antenómeros I-V; 5, ápice de la antena; 6, tórax; 7, pata derecha III; 8, uña y unguículo. Escala de la barra: 1,6-7 - 0.2 mm; 2-5, $8-0.05 \mathrm{~mm}$. 

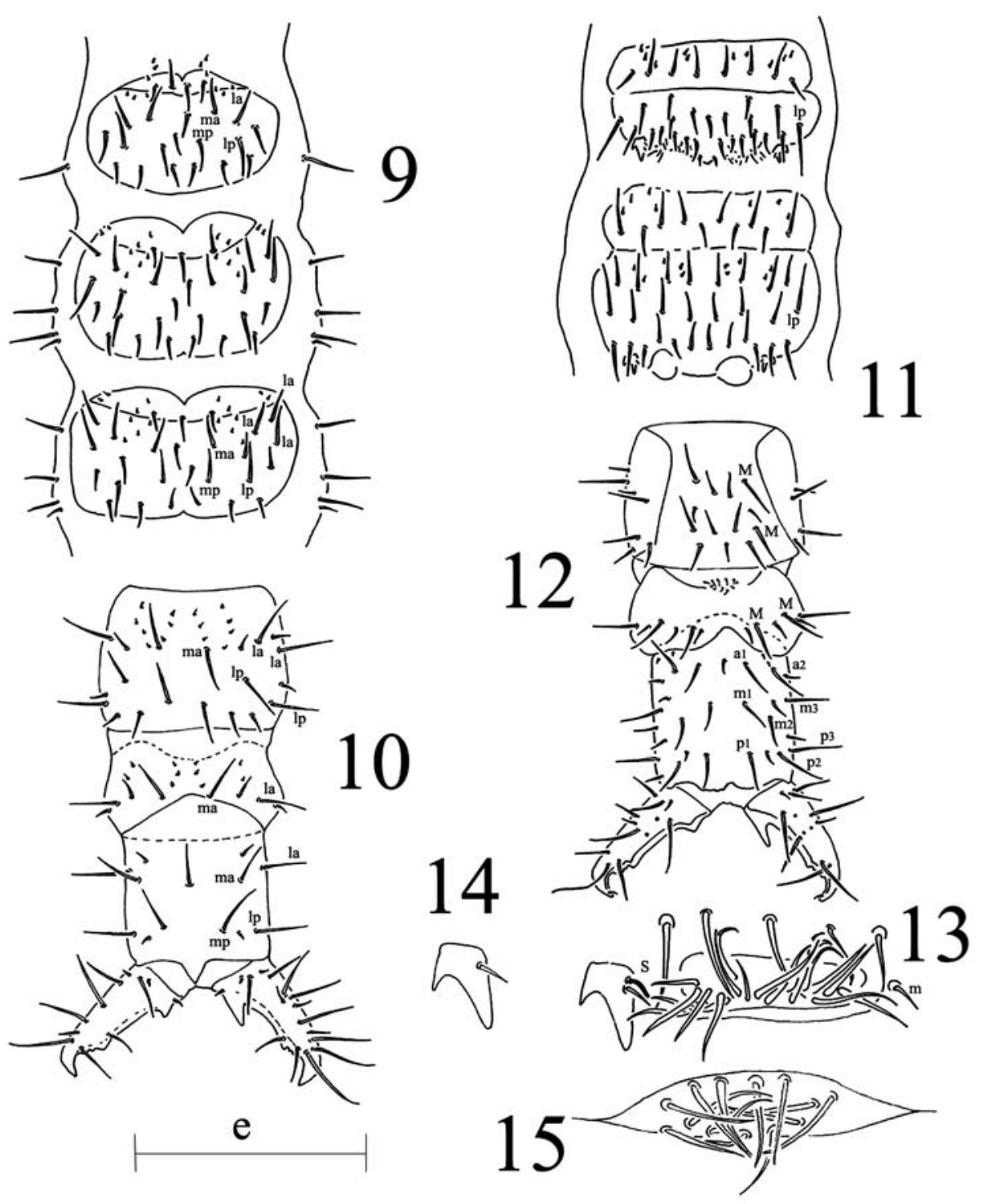

Figuras 9-15. Lacandonajapyx cristinae nov. sp. 9, abdomen I-III; 10, abdomen I-X; 11, esternito I-II; 12, Esternito VIII-X; 13, subcoxa, 14, estilo IV; 15, papila genital.

Escala de la barra: 9-12 - 0.2 mm; 13-15 - $0.05 \mathrm{~mm}$. 
Esternito I (Fig. 11).

Prescutum, 5+5 m y 4+4 n; escutum, 1+1 M (lp), 10+1+10 n.

Órgano subcoxal (Fig. 13): al igual que en Parajapyx dorianus, la subcoxa abarca la misma área posterior, con un total de 18 sedas, en la parte externa, hacia el centro del cuerpo, se presenta $1 \mathrm{~m}$. Sin órgano glandular medio.

Esternito II (Fig. 11) al VII; Prescuto, 4+4 m, 4-5+1+5-4 sedas; escuto, 5+5 m, 1+1 M (lp), 13+13 n. Esternito VIII (Fig 12). 2+2 M, 4+1+4 n. Esternopleurito IX. 3+3 M además de $3+3 \mathrm{n}$. Esternito $X .2+2 \mathrm{~m}$, tres líneas de $M(2 \mathrm{a}, 3 \mathrm{~m}, 3 \mathrm{p}), 5-6+1+6-5 \mathrm{n}$.

Vesículas eversibles: en los esternitos II-III.

Estilos: en los segmentos I-III, donde presenta 1 pequeña sensila acompañada de $1 \mathrm{~m}$ (Fig. 13), los restantes no la presentan (Fig. 14).

Papila genital femenina (Fig. 15): $6+1+6 \mathrm{n}$.

Cercos: unisegmentados subsimétricos sin orificio glandular.

Cerco derecho: con cuatro dientes internos, tres de ellos, casi al final del cerco; el cuarto se encuentra en la parte basal de gran tamaño, aproximadamente cinco veces más grande que los anteriores. Dorsalmente (Fig. 10) $3 \mathrm{~m}$ en la base del cerco; $8 \mathrm{M}$ y $5 \mathrm{n}$. Ventralmente (Fig. 12) $2 \mathrm{~m}$ basales más una distal, $7 \mathrm{M}$ y $2 \mathrm{n}$.

Cerco izquierdo: con cuatro dientes internos, misma forma que los anteriores excepto el cuarto, el cual es una tercera parte más pequeño que el derecho. Dorsalmente (Fig. 10) con $3 \mathrm{~m}$ anteriores, $7 \mathrm{M}, 1 \mathrm{n}$. Ventralmente (Fig. 12) $3 \mathrm{~m}$, una en la punta del cerco, $4 \mathrm{M}$ y $4 \mathrm{n}$.

Afinidades. Lacandonajapyx critinae nov. sp. se diferencia de Parajapyx dorianus (Silvestri 1929), por la presencia de una seda adicional en el pronoto, ausencia de $1 \mathrm{M}$ (ma) del mesoescutum, y presencia de tres $\mathrm{M}$ en el cerco derecho y dos en el izquierdo.

Datos de colecta. 24.X.2004, México, Chiapas Municipio de Ocosingo. Reserva Comunal Sierra de la Cojolita, frente al área natural protegida de Lacandonia schismatica, $16^{\circ} 44^{\prime} 53.1^{\prime \prime} \mathrm{N}$; $91^{\circ} 01^{\prime} 03^{\prime \prime}$ W. $209 \mathrm{~m}$ snm. 5 hembras ex hojarasca. R. Paredes Col.

Tipos. Holotipo 우, paratipos, 4 우우, depositados en el Laboratorio de Ecología y Sistemática de Microartrópodos de la Facultad de Ciencias, Universidad Nacional Autónoma de México (LESM-FC-UNAM).

Etimología: la especie está dedicada a la Señora Cristina Sánchez, quien fue la persona más importante en la formación académica del autor.

\section{DISCUSIÓN Y CONCLUSIONES}

En México los parajapígidos han sido poco estudiados, registrándose únicamente Parajapyx maxicanus, en el estado de Yucatán (Paclt 1957), y el género Miojapyx para el norte de México (Pagés 1989). Lo que hace este nuevo registro importante para la distribución de la familia, ya que al encontrarla en los dos extremos del país, 


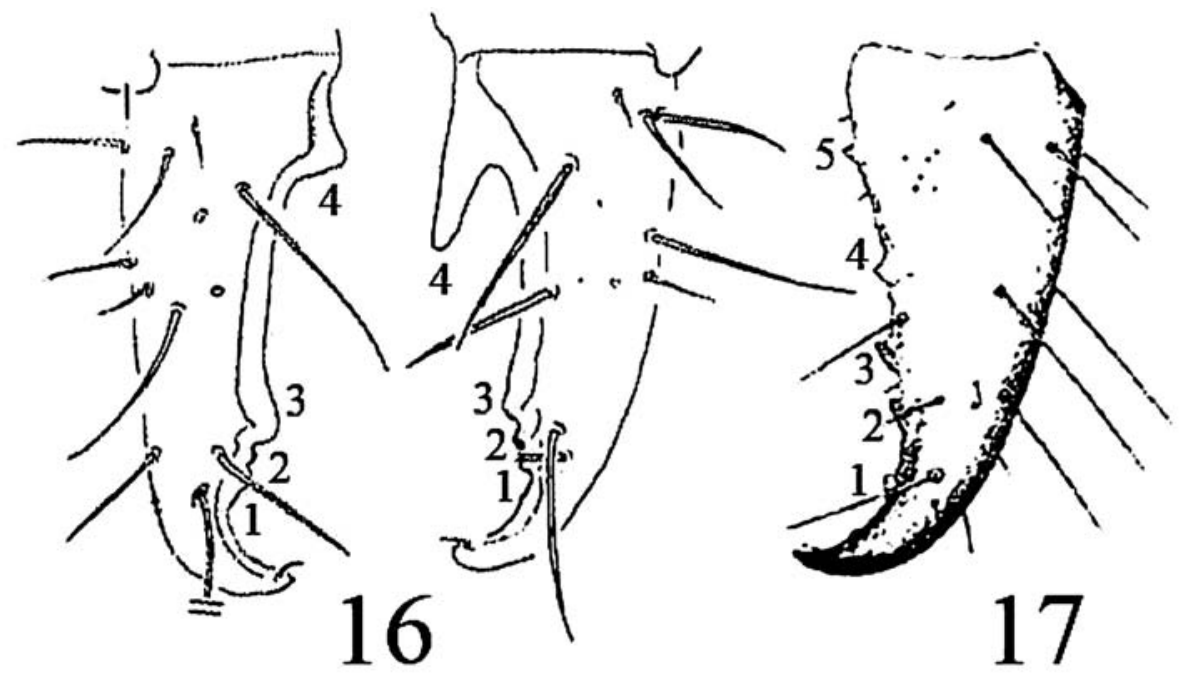

Figuras 16-17; 16. cerco de Lacandonajapyx cristinae con cuatro dientes internos:

17 cerco de Parajapyx vinciguerranus con cinco dientes internos (Tomado de Silvestri, 1929).

es factible que a lo largo y ancho del mismo podamos encontrar especies de alguno de los géneros, sobre todo de Miojapyx, del cual no se ha encontrado organismos, ni el área tipo de dicho taxón.

De forma general, para la colecta de dipluros, Pagés (1967) ha mencionado que se pueden encontrar en cualquier época del año, pero a lo largo del mismo pueden desplazarse a sitios con diferentes porcentajes de humedad, de tal forma que su captura no es continua, por lo cual pueden desaparecer frecuentemente. Por lo tanto, de las dos visitas realizadas (marzo y octubre del 2004), solo se les localizó en octubre; pero si a esto le sumamos que es una zona protegida, como lo es la Selva Lacandona, la obtención de organismos se complica aún más.

Por otro lado, los japigidos son poco abundantes y de hábitos territoriales, lo que hace raro encontrar dos organismos cercanos, principalmente de machos. Éstos, para reproducirse dejan el espermatóforo y segregan feromonas para atraer a la hembra y alejarse entre ellos (Pagés \& Baeth 1994), aunque este comportamiento se ha visto principalmente en Japyx, es muy probable que en Lacandonajapyx se presente algo parecido, por ello la falta de machos en la colecta realizada.

El nuevo género presenta un conjunto de caracteres (Cuadro 3), de los cuales el cerco de cuatro dentículos (Fig. 16) es importante. Sin embargo, Pagés (1989) menciona a Parajapyx con un cerco terminal generalmente de cinco dientes (Fig. 17.), encontrando tres excepciones, dos especies con cuatro y una con seis, por lo 
tanto dicho carácter no es consistente. Se hace mención que los dientes derechos presentan la misma forma, en comparación a los izquierdos, exceptuando a $P$. dorianus quien presenta cinco dientes internos, siendo el basal de forma diferente.

Cuadro 3. Diferencias diagnosticas de los cuatro géneros de Parajapygidae.

\begin{tabular}{lcccc}
\hline & Parajapyx & Ectasjapyx & Miojapyx & Lacandonajapyx \\
\hline Mandíbula & & & & \\
$\quad$ dientes & 5 & 4 & $?$ & 5 \\
$\quad$ dentículos & 4 & 3 & $?$ & 3 \\
Maxilas & 2 & 2 & ausentes & presentes \\
lamelas & presentes & presentes & si & no \\
Vesículas en el abdomen II-III & no & si & $?$ & no \\
Abdomen VII y IX mismo tamaño & si & $?$ & 5 & 4 \\
Orifico glandular cerca del cerco & $4-6$ & 5 & & \\
Dientes internos del cerco & & & & \\
&
\end{tabular}

Al comparar los dentículos y las lamelas se observan diferencias de dichas estructuras en cada género, esto es importante ya que el número de dientes y dentículos separan géneros (Paclt 1957), de tal forma se pueden contemplar como estructuras consistentes.

Otra estructura importante es el poro glandular ceca de los cercos, el cual está presente en Parajapyx, sin embargo, dicha estructura no aparece en los ejemplares revisados, tomándose como carácter importante para el nuevo género descrito.

Finalmente se propone el número de dentículos y la ausencia del poro glandular como caracteres diagnósticos de Lacandonajapyx, según la siguiente clave.

\section{Clave de géneros de la familia Parajapygidae}

1.- Sin vesículas coxales sobre los esternitos abdominales ........Miojapyx Ewing 1941

Un par de vesículas coxales sobre los esternitos abdominales II-III . . . . . . . . . . 2 2.- Mandíbula con cuatro dientes, sin dentículos intercalados; pocas macrosedas . ........ .................................... Ectasjapyx Silvestri 1929

Mandíbula con cinco dientes, con dentículos intercalados; numerosas macrosedas . ...3 3.- Mandíbula con cinco dientes y cuatro dentículos, poro glandular cerca del cerco ...... ........................................Parajapyx Silvestri 1903

Mandíbula con cinco dientes y tres dentículos, sin poro glandular cerca del cerco ..... . Lacandonajapyx gen. nov. 
Con respecto a la descripción de la especie, se tomaron abreviaturas y estructuras mencionadas por vez primera para la familia, siendo de gran importancia para una descripción más detallada de la especie.

AGRADECIMIENTOS. Se le agradece a los Drs. Gabriela Castaño Meneses y José Palacios Vargas, la revisión del manuscrito, además al proyecto "Lacandonis schisnatica recurso genético para México y conservación de la Selva Lacandona" CONACYT No COI-043/B1, por la facilidad prestada para las colectas en el estado de Chiapas.

\section{LITERATURA CITADA}

Ferguson, L. M. 1990. Insecta: Diplura. pp 951-963. In: D. L. Dindal (ed), Soil Biology Guide. Wiley, New York.

Paclt, J. 1957. Diplura Genera Insectorum, Quatre-Bras, Crainhem, Belgique, 123 pp.

Pagés J. 1967. Données sur la biologie de Dipljapyx humberti (Grassi). Thèse de docteur ès sciences naturelles. Faculté des Sciences de 1'Université de Dijon, France.

Pagés, J. 1989. Sclérites et appendices de l'abdomen des diploures (Insecta, Apterygota). Archives des Sciences, Genève, 42: 509-551.

Pagés, J. 1996. Un Evalljapygidae (Diplura) canadien: Evalljapyx saudersi n. sp. Dicellurata Genavensia XXI. Revue Suisse de Zoologie, 103(2) 355-367.

Pagés, J \& C. Bareth. 1994. Les diploures japygides. Pp. 280-283. In: C. Juberthie et V. Decu (Eds). Encyclopaedia biospeologica. Société de Biospéologie. Moilis - Bucarest.

Silvestri, F. 1929. Nuevos Parajapiginos de África (Thys. Japygidae). Memorias de la Real Sociedad Española de Historia Natural, XV: 221-235. 